\title{
Slow-growing Intrahepatic Cholangiocarcinoma - Difficult Diagnosis, Prolong Survival
}

\author{
Sorin Alexandrescu ${ }^{1,2}$, Razvan Grigorie ${ }^{1}$, Mirela Boros ${ }^{1}$, Vlad Herlea ${ }^{3,4}$, Irinel Popescu ${ }^{1,4}$
}

Corresponding author:

Razvan Grigorie, MD, PhD

Dan Setlacec Centre of General

Surgery and Liver Transplantation

Fundeni Clinical Institute, Soseaua

Fundeni, 258, sector 2, Bucharest

Romania

Tel/Fax: +40213180417

Email: mihai.grig@gmail.com

\author{
Abbreviations: \\ ICC - Intrahepatic cholangiocarcinoma \\ HCC - hepatocellular carcinoma \\ $\mathrm{MRI}$ - magnetic resonance imaging \\ CT - computed tomography \\ HE- Haematoxylin Eosin
}

\author{
'Dan Setlacec Centre of General Surgery and Liver Transplantation, Fundeni Clinical Institute, \\ Bucharest, Romania \\ 2University of Medicine and Pharmacy Carol Davila, Bucharest, Romania \\ ${ }^{3}$ Department of Pathology, Fundeni Clinical Institute, Bucharest, Romania \\ ${ }^{4}$ Titu Maiorescu University, Bucharest, Romania
}

\section{ABSTRACT}

Intrahepatic cholangiocarcinoma (ICC) is usually diagnosed in advanced stages of disease, more than half of the patients being unresectable at the time of their diagnosis. Moreover, the disease is very aggressive, almost half of untreated patients dying during the first 4 month after diagnosis. Even in patients who undergo curative-intent treatment, the prognosis is still modest, with median survival less than 30 months in most series. Although this dismal prognosis is typical for ICC, some patients experience unusual long-term survival, even when poor clinico-pathologic prognostic factors are present. A 59 year-old female presented on CT scan and MRI an equivocal lesion, refusing biopsy for two years. During this period the tumor doubled its diameter and the patient eventually accepted to undergo percutaneous CT-guided biopsy. Pathologic evaluation of the specimen and immunohistochemistry assay suggested an ICC. Subsequently, the patient underwent curative-intent surgery, being alive, without evidence of disease at 10 years after operation. A discussion about diagnosis and prognostic factors related to overall survival and recurrence after curative-intent surgery is carried out, emphasizing the emerging role of molecular biomarkers.

Key words: intrahepatic cholangiocarcinoma, liver resection, delayed diagnosis, survival, slow-growing

\section{INTRODUCTION}

Intrahepatic cholangiocarcinoma (ICC) is typically diagnosed in an advanced state of disease and the survival is usually short, half of untreated patients dying within 3-4 months of presentation (1). Even after curative-intent surgery, the survival rates are lower than those reported in other liver malignancies (e.g. colorectal liver metastases, hepatocellular carcinoma). Thus, the median survival after liver resection of ICC is usually less than 30 months and 5-year overall survival rates are up to $30 \%$ in most series (2). Because of the low survival rates reported even after curative-intent hepatectomy, ICC is considered a very aggressive malignancy, whose prognosis seems to be improved if liver resection is performed as soon as possible after diagnosis. 
Sometimes, the diagnosis is not easily established by dynamic investigations like CT scan or MRI and these patients confront initially with equivocal diagnosis. In such instances, the percutaneous CT-guided biopsy is useful to establish the diagnosis.

Despite of these observations, the current paper presents the case of a 59 year-old patient who was operated more than 2 years after the initial observation of the tumor. Even in the context of this prolonged observation interval, the outcome of the patient was very good, being alive, without evidence of disease at 10 years after operation. The difficulties to establish the diagnosis and the prognostic factors associated with survival after resection of ICC will be further presented.

\section{CASE REPORT}

A 59 year-old female-patient, who underwent open cholecystectomy for gallbladder lithiasis in 1994, was investigated for minor pain in the right upper quadrant in 2006. Abdominal ultrasonography raised the suspicion of a small liver mass $(2.9 \mathrm{~cm})$ in the right hemiliver, recommending the performance of a contrastenhanced abdominal CT scan. The CT scan revealed a 3 $\mathrm{cm}$. liver mass centrally-located (segments 4-5), without radiologic features of malignancy, suggesting a focal nodular hyperplasia without central scar. A contrast-enhanced MRI was subsequently performed, revealing a $3-\mathrm{cm}$ lesion adjacent to the right portal vein and right bile duct, extended in the liver parenchyma of segments 4-5, without right-sided intrahepatic bile ducts dilation or right portal branch stenosis or thrombosis (fig. $1 a, b$ ). The peripheral enhancement of the lesion during the arterial phase and the central enhancement in the late phase of the investigation suggest an important amount of fibrotic tissue inside the lesion. In the meantime, a colonoscopy and an upper endoscopy were performed, without revealing any abnormalities. Also, the serum levels of CEA and CA 19-9 were within normal range. In such context, a CT-guided biopsy of the tumor was recommended, but the patient refused to perform the investigation. Twoyears later (in 2008), although the clinical status of the patient remained well, a CT-scan revealed the increase of the above-mentioned lesion's diameter to $6 \mathrm{~cm}$, with extension through segments 1 and 4 and encasement of the right portal branch and right bile duct, without cholestasis or portal thrombosis (fig. $2 a$ ), but presenting mild dilatation of segment 6 bile duct (fig. $2 b$ ). The serum levels of CEA and CA 19-9 were also normal. Due to the tumor growth, the patient accepted to undergo a percutaneous CT-guided tumor biopsy. The pathologic evaluation of the specimen revealed abundant fibrous stroma with islands of carcinoma cells (fig. 3, 4). Moreover, the immunohistochemistry assay revealed CK-7 positive and CK-20 negative tumor cells, suggesting an ICC. Thus, on September $8^{\text {th }}, 2008$ the patient underwent surgery. Intraoperative exploration revealed a $6 \mathrm{~cm}$ tumor, centrally-located in the liver (involving segment 5, 6 and extended to segments 4 and 1) surrounding the right glissonean pedicle and adjacent to the bile duct confluence and inferior vena cava (IVC). It was performed a right trisectionectomy extended to segment 1, lateral resection of the IVC with cavorrhaphy, common bile duct resection with Roux en-Y cholangiojejunostomy and lymphadenectomy of the hepatoduodenal ligament. Postoperatively, the patient developed grade $\mathrm{B}$ bile leak being discharged with a drain which was removed 6 weeks later (fig. 5). Pathologic evaluation revealed a moderate differentiated (G2) carcinoma, mass-forming morphological type with important intratumoral desmoplastic reaction, without
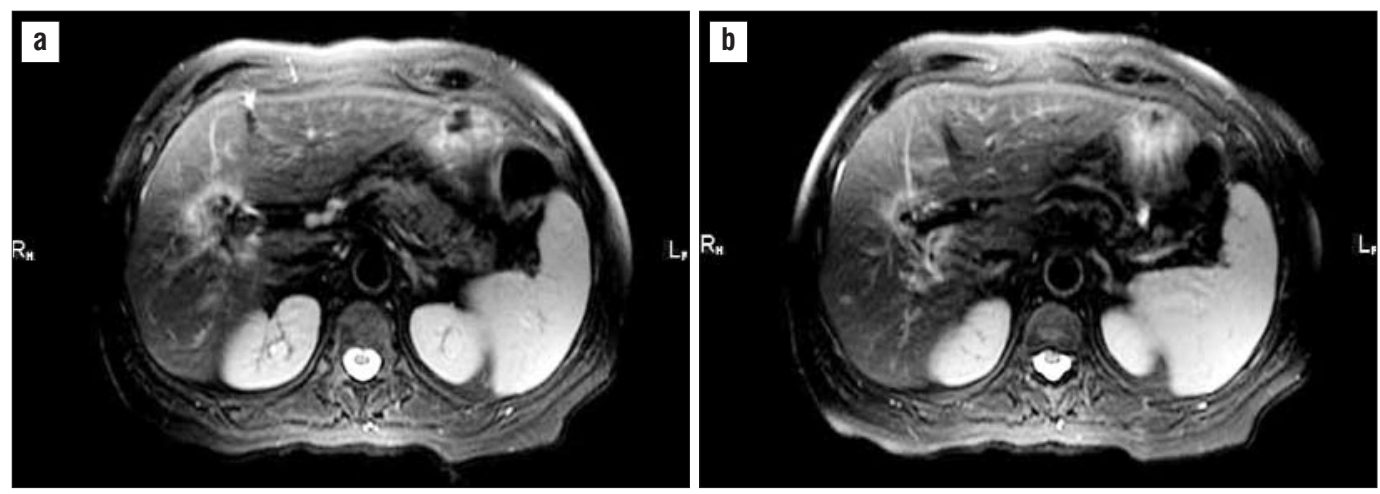

Figure 1 - MRI (June 2006) revealing a 3-cm lesion adjacent to the right portal branch (without tumor thrombus) and right bile duct (without bile duct dilatation).

a. peripheral enhancement; $b$. gradual centripetal enhancement 

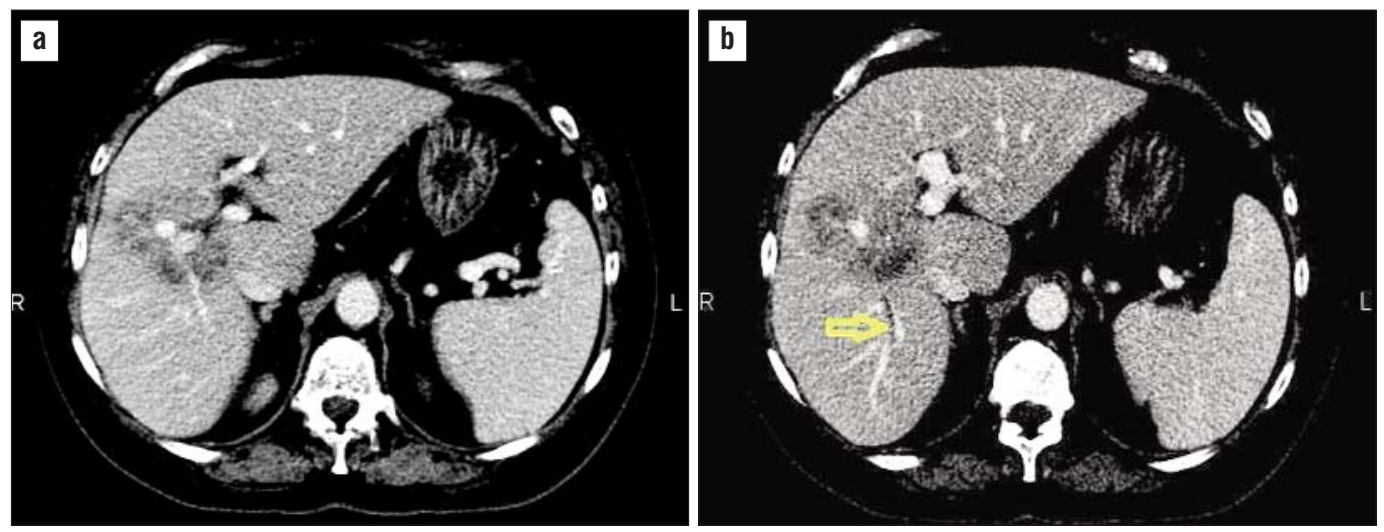

Figure 2 - CT scan (September 2008). The lesion from figure 1 which doubled its diameter during 2 years.

a. encasement of the right portal branch, without tumor thrombus; b. segment 6 bile duct dilatation (arrow)

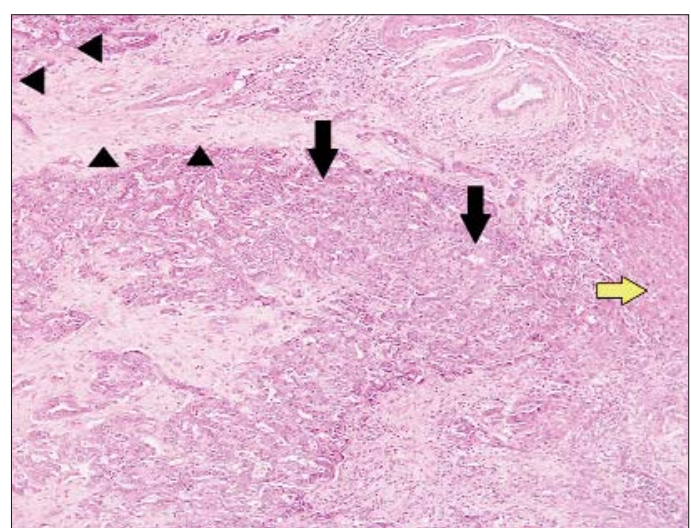

Figure 3 - HE staining (10x) of the resected specimen. Mass forming ICC with important desmoplastic reaction (arrow heads), carcinoma tissue (black arrows) and normal liver parenchyma (yellow arrow)

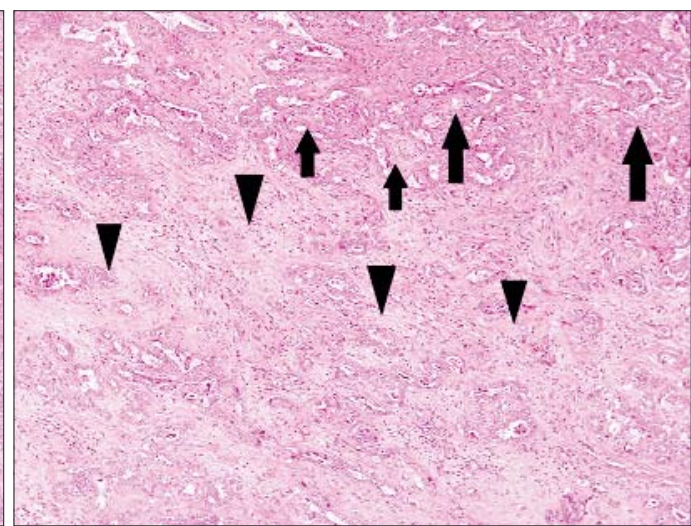

Figure 4 - HE staining (10x) of the resected specimen. Fibrous stroma (arrow heads) and carcinoma - type malignant proliferation (arrows)

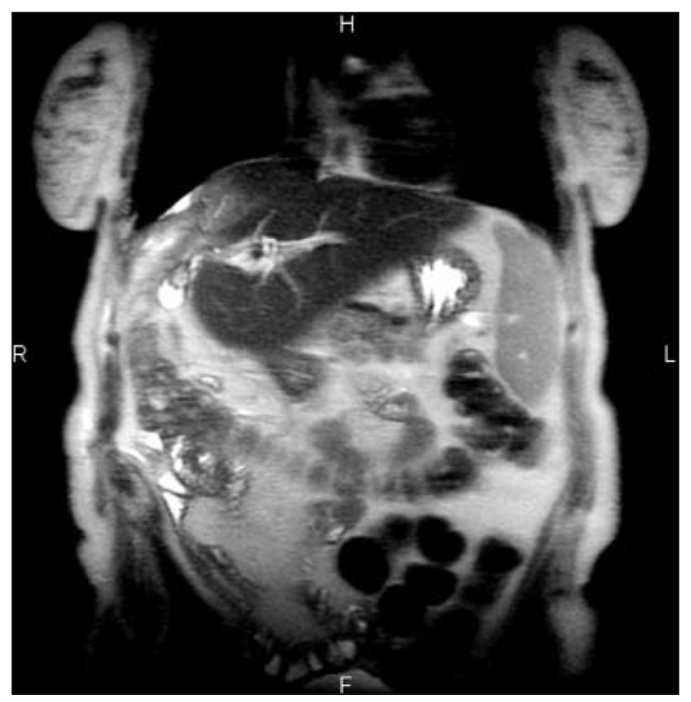

Figure 5 - MRI (January 2009). Normal findings of the remnant liver (segments 2-3) and of the Roux-en-Y cholangio-jejunostomy (3 months postoperatively) intrahepatic vascular invasion. The resection margins were larger than $1 \mathrm{~cm}$ and one lymph node presented metastasis. According to the 8th edition of the AJCC staging system for ICC, the postoperative stage would be pT1bN1M0. From November 2008 until March 2009 the patient received 6 cycles of 5 -fluorouracil and cisplatin. In present, 10 years after surgery, the patient is in good clinical condition, without evidence of recurrence (fig. 6).

Discussion. Intrahepatic cholangiocarcinoma (ICC) is the second most common primary liver malignancy after hepatocellular carcinoma (HCC), with an increasing incidence worldwide in the last decades (3)

Sometimes, the diagnosis of ICC is difficult, because the dynamic imaging methods are not similarly specific in detection of ICC, as they are for HCC diagnosis. Thus, contrast-enhanced CT scan or MRI are specific enough for HCC diagnosis in most patients, allowing treatment 


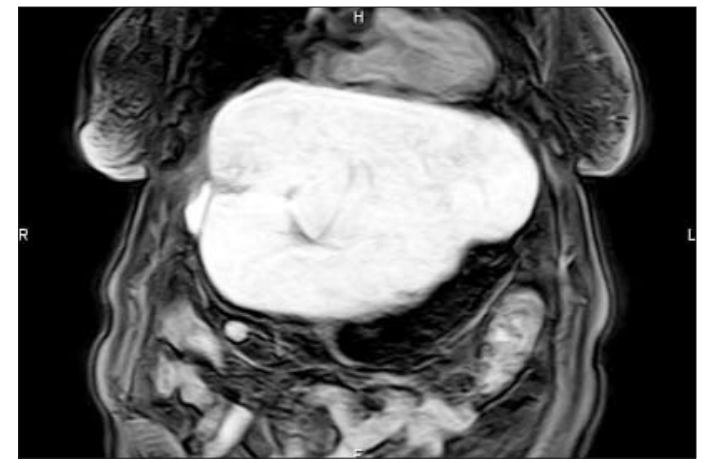

Figure 6 - MRI (February 2018). Important hypertrophy of the remnant liver, without tumor recurrence (10 years postoperatively)

allocation based on radiologic findings, without the need for biopsy (4).

These radiologic methods are not similarly specific for ICC diagnosis, mainly because the radiologic features are correlated with the morphologic (sub)types. By this reason, not all the ICCs exhibit typical imaging findings, mimicking a variety of tumoral and non-tumoral lesions (5). Therefore, sometimes, the diagnosis may be established only by the tumor biopsy. Finding an adenocarcinoma at biopsy sample should indicate that a diagnosis of cholangiocarcinoma is possible, particularly in the absence of another obvious primary lesion outside the liver (1). In the present case, the radiologic findings (from 2006) were not quite typical, due to the lack of bile duct dilatation, no evidence of bile duct stenosis and the lack of capsular retraction. On the other hand, the lesion presented irregular peripheral enhancement with gradual centripetal augmentation and vascular encasement without tumor thrombus, which are radiologic features frequently observed in mass-forming ICC (5). However, the patient was asked to perform tumor biopsy, but she refused initially (in 2006). Two years later, the imagistic re-evaluation revealed that lesion's diameter doubled over this time period and she eventually accepted the biopsy. The pathologic evaluation suggested an ICC, revealing abundant fibrosis and scattered cells susceptible of malignancy. However, the pathologic examination cannot reveal pathognomonic features for ICC, whose diagnosis is often revealed by a immunohistochemistry panel including CK7 postive staining and CK20 negative staining. Other immunohistochemistry patterns that may be suggestive of ICC include negative TTF1, CDX2, DPC4 and positive AE1/AE3 (6).

The most surprising fact was the finding that the tumor was still resectable after more than two years of surveillance, in a disease which is usually very aggressive, with nearly no survivors at three years after diagnosis, in non-resected patients (6). That slow-growing pattern of the ICC could represent the hallmark of a favorable biologic behavior, explaining the long-term survival observed in this patient. Therefore, this two years delay between the appearance of the tumor and its resection could be seen as a "test of time" that was postulated to predict biologic aggressiveness of liver metastases in patients with colorectal cancer (7). The slow-growing pattern of the tumor seems to be one of the few favorable prognostic factors present in this patient, alongside with the lack of vascular invasion, the absence of multiple tumors and R0 resection (8). By contrary, the patient presented several factors usually associated with dismal prognosis. For example, tumor size larger than $5 \mathrm{~cm}$ and the presence of lymph node metastases are associated with increased risk of tumor recurrence in patients who underwent curative-intent surgery for ICC (9). Moreover, a recently published paper revealed that the development of postoperative complications represented an independent predictor of worse long-term outcome in patients with resected ICC (10). However, the negative impact of lymph node metastases might be balanced by the performance of lymphadenectomy, which seems to improve survival, according to Kim et al (11).

Another explanation for the favorable outcome of this patient, despite the above-mentioned negative prognostic factors, may be the less aggressive behavior of the tumor, which was indirectly revealed by its slowgrowth. For better understanding the biologic behavior of ICC, future identification of prognostic bio-markers seems to be of tremendous importance. This finding suggests that molecular prognostic factors could be more sensitive to predict long-term outcomes in patients undergoing curative-intent hepatectomy for ICC, than clinico-pathological factors that are used in present. Thus, a recent study revealed that the presence of FGFR2 translocation in ICC was associated with statistically significant higher median survival after surgery (12), whereas KRAS-mutant ICCs are associated with higher incidence of R1 resection rates (13).

In summary, when dynamic radiologic investigations cannot establish the diagnosis of a liver lesion, biopsy is mandatory, to avoid the progression to unresectability of a potential malignant tumor. Even in patients with locally advanced ICCs, presenting unfavorable clinico-pathologic prognostic factors, curative-intent liver resection could offer good longterm outcomes. Future identification of molecular biomarkers might allow a better selection of patients for liver resection. 


\section{Conflicts of interest}

The authors declare that there are no conflicts of interest.

\section{REFERENCES}

1. Patel, T. Cholangiocarcinoma--controversies and challenges. Nat Rev Gastroenterol Hepatol. 2011;8(4):189-200.

2. Spolverato G, Kim Y, Alexandrescu S, Popescu I, Marques HP, Aldrighetti L, et al. Is Hepatic Resection for Large or Multifocal Intrahepatic Cholangiocarcinoma Justified? Results from a MultiInstitutional Collaboration. Ann Surg Oncol. 2015;22(7):2218-25.

3. Aljiffry M, Abdulelah A, Walsh M, Peltekian K, Alwayn I, Molinari M. Evidence-based approach to cholangiocarcinoma: a systematic review of the current literature. J Am Coll Surg. 2009;208(1):134-47.

4. Gupta M, Gabriel H, Miller FH. Role of Imaging in Surveillance and Diagnosis of Hepatocellular Carcinoma. Gastroenterol Clin North Am. 2018;47(3):585-602

5. Chung YE, Kim MJ, Park YN, Choi JY, Pyo JY, Kim YC, et al. Varying appearances of cholangiocarcinoma: radiologic-pathologic correlation. Radiographics. 2009;29(3):683-700.

6. Dodson RM, Weiss MJ, Cosgrove D, Herman JM, Kamel I, Anders $\mathrm{R}$, et al. Intrahepatic cholangiocarcinoma: management options and emerging therapies. J Am Coll Surg. 2013; 217(4):736-750.e4.
7. Malik HZ, Farid S, Al-Mukthar A, Anthoney A, Toogood GJ, Lodge JP, et al. A critical appraisal of the role of neoadjuvant chemotherapy for colorectal liver metastases: a case-controlled study. Ann Surg Oncol. 2007:14(12):3519-26.

8. de Jong MC, Nathan H, Sotiropoulos GC, Paul A, Alexandrescu S, Marques $\mathrm{H}$, et al. Intrahepatic cholangiocarcinoma: an international multi-institutional analysis of prognostic factors and lymph node assessment. J Clin Oncol. 2011;29(23):3140-5.

9. Hyder 0, Hatzaras I, Sotiropoulos GC, Paul A, Alexandrescu S, Marques $\mathrm{H}$, et al. Recurrence after operative management of intrahepatic cholangiocarcinoma. Surgery. 2013;153(6):811-8.

10. Spolverato G, Yakoob MY, Kim Y, Alexandrescu S, Marques HP, Lamelas J, et al. Impact of complications on long-term survival after resection of intrahepatic cholangiocarcinoma: Impact of Morbidity on Long-Term Outcomes of ICC. Cancer. 2015; 121(16):2730-9.

11. Kim SH, Han DH, Choi GH, Choi JS, Kim KS. Oncologic Impact of Lymph Node Dissection for Intrahepatic Cholangiocarcinoma: a Propensity Score-Matched Study. J Gastrointest Surg. 2018 Aug 15. doi: 10.1007/s11605-018-3899-2. [Epub ahead of print]

12. Graham RP, Barr Fritcher EG, Pestova E, Schulz J, Sitailo LA, Vasmatzis $\mathrm{G}$, et al. Fibroblast growth factor receptor 2 translocations in intrahepatic cholangiocarcinoma. Hum Pathol. 2014;45(8):16308. doi: 10.1016/j.humpath.2014.03.014. Epub 2014 Apr 13.

13. Zhu AX, Borger DR, Kim Y, Cosgrove D, Ejaz A, Alexandrescu S, et al. Genomic profiling of intrahepatic cholangiocarcinoma: refining prognosis and identifying therapeutic targets. Ann Surg Oncol. 2014;21(12):3827-34. 